\title{
Dielectric hysteresis of undoped and dysprosium doped ferroelectric potassium vanadate
}

\author{
N B PATIL, R T PATIL ${ }^{1}$ and S H CHAVAN ${ }^{2 *}$ \\ Vivekanand College, Kolhapur 416002 , India \\ ${ }^{1}$ Bhogawati Mahavidyalaya, Kurukali 416 001, India \\ ${ }^{2 *}$ Department of Physics, Shivaji University, Kolhapur 416 004, India \\ MS received 30 September 1988; revised 2 January 1989
}

\begin{abstract}
Dielectric hysteresis properties of undoped and dysprosium-doped potassium vanadate have been studied in the temperature range covering their transition points. The coercive field of these materials was measured by the hysteresis loop method. It is observed that the coercive field of $\mathrm{KVO}_{3}$ doped with $\mathrm{Dy}_{2} \mathrm{O}_{3}$ at different concentrations $(0$ to $3 \mathrm{~mol} \%$ ) is remarkably dependent on doping concentration. It is also seen that undoped $\mathrm{KVO}_{3}$ shows ferroelectric behaviour up to $320^{\circ} \mathrm{C}$ while $\mathrm{Dy}_{2} \mathrm{O}_{3}$-doped $\mathrm{KVO}_{3}$ samples show ferroelectric behaviour up to $380^{\circ} \mathrm{C}$ for all concentrations.
\end{abstract}

Keywords. Ferroelectricity; hysteresis doping; coercive field; phase transition.

\section{Introduction}

Dielectric hysteresis is an essential feature of ferroelectric materials, and one of the important parameters defining hysteresis is the coercive field, which is the most sensitive property of ferroelectric materials. Wieder (1955) has defined coercivity as the magnitude of the field necessary for switching of the polarization along the major hysteresis loop. The theory of the coercive field of $\mathrm{BaTiO}_{3}$ was reported by Janovec (1958). Sawada et al (1961) discussed the temperature and amplitude dependence of the coercive field for $\mathrm{KNO}_{3}$ crystals. Bertaut and Lissalde (1967) measured the hysteresis loops in ferroelectrics by X-ray intensities and true coercive fields in yttrium and rare earth manganates. A new family of oxide ferroelectrics, $\mathrm{RVO}_{4}[\mathrm{R}=\mathrm{Nd}, \mathrm{Eu}, \mathrm{Gd}, \mathrm{Tb}, \mathrm{Dy}, \mathrm{Ho}, \mathrm{Er}, \mathrm{Yb}, \mathrm{Lu}$ and $\mathrm{Sc}]$, was discovered by Ismailzade et al (1981). Glazer et al (1984) described a new type of ferroelectric loop tracer which allows continuous compensation for stray capacitances and resistive losses of the sample. The pyroelectric and dielectric properties of ferroelectric $\mathrm{NaVO}_{3}, \mathrm{KVO}_{3}$ and their solid solutions were investigated by Patil et al (1988).

The effect of Dy-doping on the properties of $\mathrm{BaTiO}_{3}$ ceramics were investigated by Yamaji et al (1977). The dependence of permittivity of Gd-doped $\mathrm{BaTiO}_{3}$ on doping concentration was reported by Issa et al (1984). In recent years considerable advances have been made in investigations on various properties of ferroelectric $\mathrm{NaVO}_{3}$ and $\mathrm{KVO}_{3}$, but the effect of impurities on these properties have practically not been studied thus far.

The objective of the present paper is to report the behaviour of the dielectric hysteresis of undoped $\mathrm{KVO}_{3}$ and that doped with different concentrations of $\mathrm{Dy}_{2} \mathrm{O}_{3}(0.025$ to $3 \mathrm{~mol} \%)$ and to study the variation of the coercive field with temperature and doping concentration for these ceramic materials.

\footnotetext{
*For correspondence.
} 


\section{Experimental}

The polycrystalline solid of potassium vanadate was prepared from a stoichiometric mixture of $\mathrm{K}_{2} \mathrm{CO}_{3}$ of purity $99 \%$ (K Chem. Chemicals) and $\mathrm{V}_{2} \mathrm{O}_{5}$ of purity $>99 \%$ [Fluka A G, Switzerland], according to the reaction

$$
\mathrm{K}_{2} \mathrm{CO}_{3}+\mathrm{V}_{2} \mathrm{O}_{5}=2 \mathrm{KVO}_{3}+\mathrm{CO}_{2} \uparrow \text {. }
$$

The mixture was slowly heated in a platinum crucible inside a globar furnace up to $750^{\circ} \mathrm{C}$ for $5 \mathrm{~h}$ and then allowed to cool up to room temperature. The Dy additive used was $\mathrm{Dy}_{2} \mathrm{O}_{3}$ of $99.9 \%$ purity (John Baker Inc., Colorado, USA). The samples were prepared by weighing $\mathrm{Dy}_{2} \mathrm{O}_{3}$ in different quantities ranging from 0.025 to $3 \mathrm{~mol} \% \mathrm{Dy}_{2} \mathrm{O}_{3}$ in $\mathrm{KVO}_{3}$. Every batch was dry-mixed and then mixed wet with ethyl alcohol in an agate mortar. After complete evaporation of alcohol, the batches were calcined in a platinum crucible at $750^{\circ} \mathrm{C}$ for $5 \mathrm{~h}$ inside a globar furnace and then allowed to cool $\$$ room temperature. The samples formed were confirmed by scanning them on an X-ray diffractometer. Pellets of the samples in the form of a disc $(1 \mathrm{~cm}$ dia and about $0.1 \mathrm{~cm}$ thick) were pressed at $6 \mathrm{t}$ pressure. These pellets possessed about $90 \%$ of the theoretical density and were further sintered on platinum foil at $500^{\circ} \mathrm{C}$ for $3 \mathrm{~h}$ in a furnace. The two faces of the pellet were wellpolished and coated with a very thin layer of silver paste in order to achieve good electrical contact with the electrodes.

The experimental set-up consists of a digital micro-voltmeter and a furnace. The well-known circuit of Sawyer and Tower (1930) was used for obtaining the dynamic hysteresis loop. Pellets of $\mathrm{KVO}_{3}$ doped with different concentrations of $\mathrm{Dy}_{2} \mathrm{O}_{3}(0$, $0 \cdot 025,0 \cdot 05,0 \cdot 1,0 \cdot 5,1$ and $3 \mathrm{~mol} \%$ ) were placed in a sample holder fabricated in our laboratory and were heated slowly inside a furnace. A dielectric hysteresis loop was observed on the screen of an oscilloscope by using the $50 \mathrm{~Hz}$ a.c. electric field for a field strength of $1 \mathrm{Kv} / \mathrm{cm}$. The half-width of the hysteresis loop is taken as the coercive field at various temperatures, by calibrating the screen of the oscilloscope.

\section{Results and discussion}

The ferroelectric coercive field $(E c)$ as a function of temperature for $\mathrm{KVO}_{3}$ doped with different concentrations of $\mathrm{Dy}_{2} \mathrm{O}_{3}$ is shown in figure 1. As seen from figure 1, the coercive field $(E c)$ strongly depends upon the temperature as well as on the doping concentration of the material. Initially the coercive field remains constant in a wide range of temperature and then decreases with further increase of temperature and vanishes at a certain temperature indicating the Curie temperature $T c$ of the material. Figure 1 also shows that the Curie temperature of undoped- $\mathrm{KVO}_{3}$ as observed by the hysteresis loop method was $320^{\circ} \mathrm{C}$ which is in good agreement with the Curie temperature reported by Chavan and Suryavanshi (1985) and Patil et al (1988b), while the Curie temperature of the $\mathrm{Dy}_{2} \mathrm{O}_{3}$-doped $\mathrm{KVO}_{3}$ ceramic material for all doping concentrations $(0.025$ to $3 \mathrm{~mol} \%)$ was $380^{\circ} \mathrm{C}$. Peak values of coercive field investigated for undoped $\mathrm{KVO}_{3}$ and that doped with $\mathrm{Dy}_{2} \mathrm{O}_{3}$ are summarized in table 1 .

Table 1 reveals that the peak value of the coercive field is maximum for 0.025 mol\% doping concentration of $\mathrm{Dy}_{2} \mathrm{O}_{3}$. As doping concentration increases from 0.025 to $3 \mathrm{~mol} \%$, the peak value of coercive field decreases. 


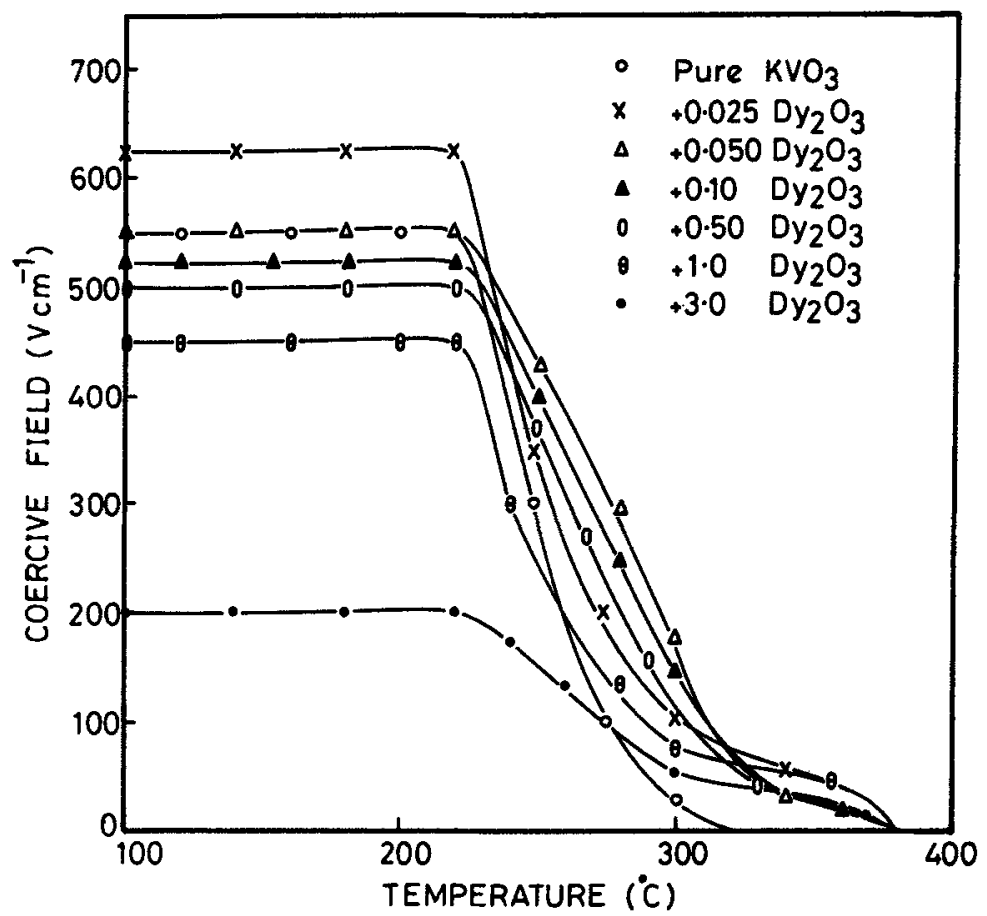

Figure 1. Variation of coercive field with temperature measured at a field of $1 \mathrm{Kv} / \mathrm{cm}$ at $50 \mathrm{~Hz}$.

Table 1. Peak values of coercive field of $\mathrm{KVO}_{3}$ with $\mathrm{Dy}_{2} \mathrm{O}_{3}$ concentration.

\begin{tabular}{lc}
\hline $\begin{array}{l}\mathrm{Dy}_{2} \mathrm{O}_{3} \text { content } \\
(\mathrm{mol} \%)\end{array}$ & $\begin{array}{c}\text { Peak value of coercive } \\
\text { field }(\mathrm{V} / \mathrm{cm})\end{array}$ \\
\hline 0 & 550 \\
0.025 & 625 \\
0.050 & 550 \\
0.10 & 525 \\
0.50 & 500 \\
1.0 & 450 \\
3.0 & 200 \\
\hline
\end{tabular}

\section{Conclusions}

(i) Undoped $\mathrm{KVO}_{3}$ ceramic material shows ferroelectric behaviour up to $320^{\circ} \mathrm{C}$, while Dy-doped $\mathrm{KVO}_{3}$ samples show ferroelectric behaviour up to $380^{\circ} \mathrm{C}$ for all concentrations $(0.025$ to $3 \mathrm{~mol} \%)$.

(ii) The coercive field of undoped $\mathrm{KVO}_{3}$ and that doped with $\mathrm{Dy}_{2} \mathrm{O}_{3}$ strongly depends upon temperature.

(iii) Peak value of the coercive field varies with doping concentrations of $\mathrm{Dy}_{2} \mathrm{O}_{3}$ in $\mathrm{KVO}_{3}$. It is maximum for $0.025 \mathrm{~mol} \%$ doping concentration and then decreases with further increase of doping concentration up to $3 \mathrm{~mol} \%$. 


\section{References}

Bertaut E F and Lissalde F 1967 Solid State Commun. 5173

Chavan S H and Suryavanshi S G 1985 Indian J. Phys. A59 555

Glazer A M, Groves P and Smith D T 1984 J. Phys. E: Sci. Instrum. 1795

Ismailzade I H, Iskenderov R N, Alekberov A I, Ismailov R M, Habibov A M and Salyev F M 1981 Ferroelectrics 3145

Issa M A A, Molokhia N M and Nasser S A 1984 J. Phys. D: Appl. Phys. 17571

Janovec V 1958 Czech. J. Phys. 83

Patil T A, Jamadar V M and Chavan S H 1988a Indian J. Phys. A62 341

Patil T A, Jamadar V M and Chavan S H 1988b Indian J. Pure Appl. Phys. 26456

Sawada S, Nomura S and Yohko A 1961 J. Phys. Soc. Jpn. 1612

Sawyer C B and Tower C H 1930 Phys. Rev. 35269

Wieder H H 1955 J. Appl. Phys. 261479

Yamaji A, Enomoto Y, Kinoshita K and Murakami T 1977 J. Am. Ceram. Soc. 6097 